\title{
Efecto de dosis de nitrógeno, fósforo y potasio sobre el crecimiento del tomate de árbol (Solanum betaceum Cav.) en etapa vegetativa
}

\section{Effect of nitrogen, phosphorus, and potassium doses on tree tomato (Solanum betaceum Cav.) growth in the vegetative phase}
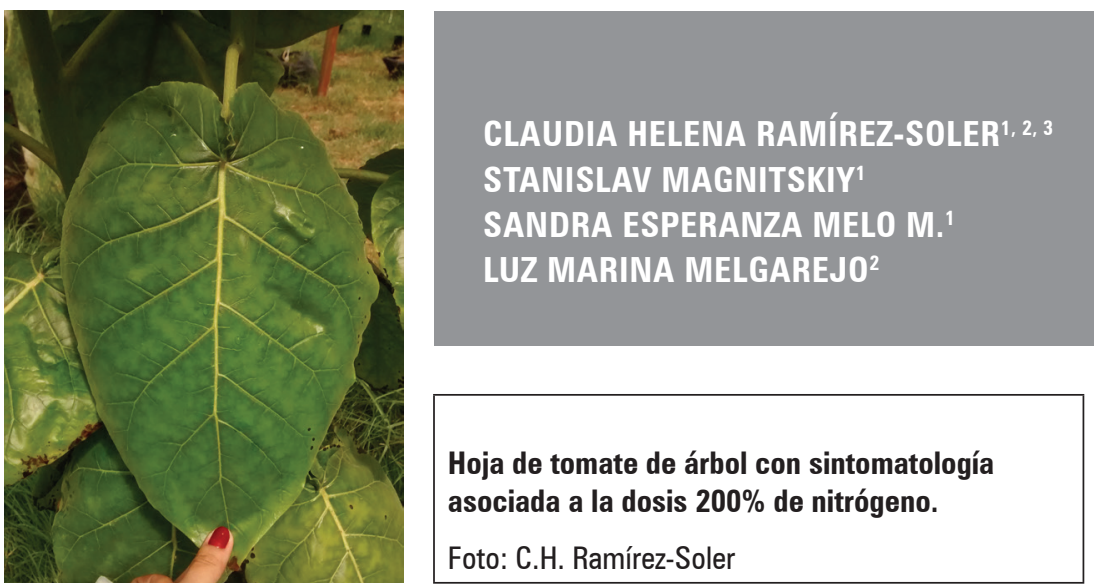

\section{RESUMEN}

El presente trabajo tuvo como objetivo evaluar el efecto de dosis de nitrógeno, fósforo y potasio sobre el crecimiento del tomate de árbol en etapa vegetativa. Se realizó bajo condiciones de invernadero en la Universidad Nacional de Colombia (sede Bogotá) y se empleó un diseño de bloques aleatorizado, donde se evaluaron ocho tratamientos, con tres réplicas, así: CC, control comercial o dosis recomendada; CS, control suelo sin ninguna fertilización; Bajo N, 10\% de la dosis recomendada (DR); Bajo P, 10\% DR; Bajo K, 10\% DR; Alto N, 200\% DR; Alto P, 200\% DR y Alto K, 200\% DR. El plan de fertilización se ajustó según análisis físico-químico del suelo y dosis reportadas para el cultivo. Para determinar el crecimiento de las plantas se emplearon algunos parámetros e índices de crecimiento utilizando un modelo logístico de enfoque funcional. Los resultados evidenciaron que el tratamiento CS y Alto P redujeron cerca de un 50\% el área foliar, número de hojas, longitud total de la planta y masa seca de las hojas a diferencia del control comercial (CC). El tratamiento Bajo K aumentó en un $70 \%$ las variables mencionadas, así como la masa seca total. Para las tasas de crecimiento el tratamiento Bajo K presentó el mayor índice de área foliar y tasa relativa de crecimiento, y el tratamiento Alto K presentó la mayor tasa de crecimiento del cultivo.

\footnotetext{
Facultad de Ciencias Agrarias, Departamento de Agronomía, Universidad Nacional de Colombia, Bogotá (Colombia). ORCID Ramírez-Soler, C.H.: 0000-0002-3283-2675; ORCID Magnitskiy, S.: 0000-0002-3715-1932; ORCID Melo M., S.E.: 0000-0002-4875-7657

2 Facultad de Ciencias, Departamento de Biología, Laboratorio de Fisiología y Bioquímica Vegetal, Universidad Nacional de Colombia, Bogotá (Colombia). ORCID Melgarejo, L.M.: 0000-0003-3148-1911

Autor para correspondencia.chramirezs@unal.edu.co

Artículo basado en la tesis de maestría de la primera autora "Respuesta fisiológica de plantas de tomate de árbol (Solanum betaceum Cav.) bajo deficiencias y diferentes niveles de nitrógeno, fósforo y potasio".
} 


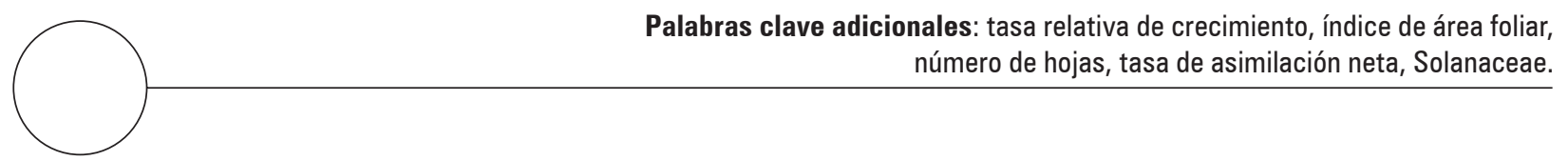

\section{ABSTRACT}

The objective of this study was to evaluate the effects of nitrogen, phosphorus and potassium doses on tree tomato (Solanum betaceum Cav.) growth in the vegetative phase. This research was conducted under greenhouse conditions at the National University of Colombia, Bogotá. A randomized block design was employed to evaluate eight treatments with three replicates as follows: i) CC: commercial control, ii) CS: control without fertilization, iii) Low N: $10 \%$ of the recommended dose (DR); iv) Low P: $10 \%$ DR; v) Low K: 10\% DR, vi) High N: 200\% DR; vii) High P: $200 \%$ DR, and viii) High K: 200\% DR. The fertilizer plan was adjusted according to the soil analysis and doses reported for the species. To determine plant growth, growth rate indexes were used based on the functional logistic model. The treatment without fertilization CS and High P reduced the leaf area, number of leaves, stem length, and dry mass of leaves of the tree tomatoes by about $50 \%$, unlike the commercial control (CC). The Low $\mathrm{K}$ treatment increased the same variables by $70 \%$, as well as the total dry mass. For growth rates, the Low $\mathrm{K}$ treatment resulted in the highest leaf area index and relative growth rate, and the High $\mathrm{K}$ treatment presented the highest crop growth rate.

Additional key words: relative growth rate, leaf area index, leaf number, net assimilation rate, Solanaceae.

Fecha de recepción: 31-10-2017 Aprobado para publicación: 30-01-2018
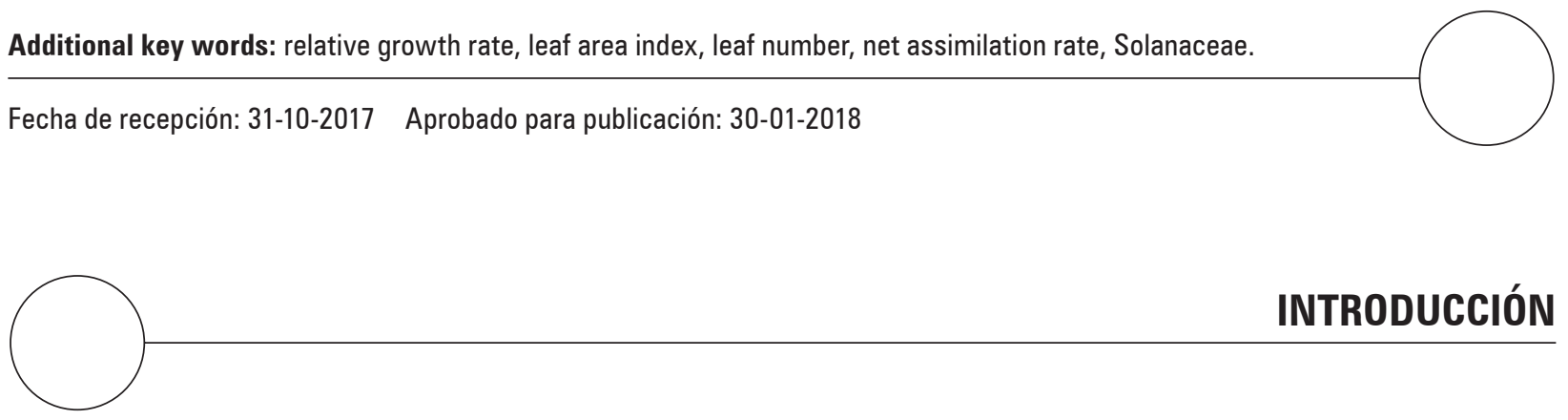

El tomate de árbol (Solanum betaceum Cav.) es originario de los bosques Andinos (Acosta-Quezada et al., 2015), es considerado parte de los cultivos con importancia en la región Andina Colombiana, a causa de su rol en la industria de la bioprospección (Osorio et al., 2012). Actualmente, su disminución en áreas de cultivo es a causa del inadecuado uso de fertilizantes (excesos y deficiencias). La información sobre nutrición en tomate de árbol es escasa; a diferencia de otras solanáceas, como papa (Rios et al., 2010), uchuva (Martínez et al., 2008) y tomate (Kanai et al., 2011), para las cuales se registran estudios sobre parámetros fisiológicos y de crecimiento generados a partir de la nutrición. De los elementos más estudiados, a causa de la mayor demanda y uso, se encuentran los macroelementos como nitrógeno $(\mathrm{N})$, fósforo $(\mathrm{P})$ y potasio (K) (Baloch et al., 2014).

Los elementos como el N, P y K son descritos como los tres factores esenciales en la nutrición vegetal (Hu et al., 2016). El N, frente a la ausencia o bajo abastecimiento de nutrientes, la fijación biológica del nitrógeno se ve limitada (Divito y Sadras, 2014); debido a que es importante para el crecimiento, capacidad fotosintética y síntesis de clorofila (Boussadia et al., 2010). El P, después del N, es el macronutriente más limitado para los cultivos, a causa de la restricción en su disponibilidad que puede ser alrededor de $40 \%$ a nivel edáfico (Vitousek et al., 2010). Además, el $\mathrm{P}$ participa en procesos como la transferencia de energía, respiración y fotosíntesis (Marschner, 2012); es constituyente del ADN y ARN, también de moléculas para transferencia energética (ADP y ATP), e interviene en la división celular (Kalaji et al., 2014). El K, es el catión inorgánico primordial que participa en procesos fisiológicos y metabólicos como la homeostasis del $\mathrm{pH}$ citoplasmático, activación enzimática, la actividad estomática, translocación de fotoasimilados y el potencial osmótico (Oosterhuis et al., 2014). Armengaud et al. (2009) indican que el $\mathrm{K}$ tiene efectos sobre la transducción de energía, la actividad de enzimas implicadas en la asimilación de amonio, el crecimiento y la conversión de aminoácidos. 
Para la producción de cultivos de importancia económica en el país, es necesario conocer los efectos de diferentes dosis de macroelementos sobre la respuesta fisiológica y el crecimiento. Actualmente, no se evidencia suficiente literatura sobre la nutrición y los parámetros fisiológicos y de crecimiento en el tomate de árbol y si se encuentran son insuficientes y desactualizados. Clark y Richardson (2002) registraron en plantas de tomate de árbol, un incremento en la acumulación de biomasa, macro y micro elementos, concluyendo que existe una relación entre la acumulación de biomasa y los elementos minerales; también, indicaron que el $\mathrm{N}, \mathrm{P}$ y K fueron los nutrientes con mayor demanda a través del tiempo en la planta. Por otro lado, Pilco (2009), utilizando diferentes formulaciones de fertilizantes comerciales en plantas de tomate de árbol, concluyó que el suministro del 100\% del fertilizante Besacote $6 \mathrm{M}^{\oplus}$ (BASF, Ecuador) (16\% $\mathrm{N}, 8 \% \mathrm{P}_{2} \mathrm{O}_{5}, 12 \% \mathrm{~K}_{2} \mathrm{O}, 2 \% \mathrm{MgO}, 5 \% \mathrm{SO}_{4}, 0,004 \% \mathrm{Fe}$, 0,05\% Cu, 0,06\% Mn, 0,02\% Zn, 0,02\% B, 0,015\% Mo), resultó en los valores más altos de área foliar, número de hojas, altura de la planta, y diámetro del tallo en la fase vegetativa. Montalvo (2010), indica que en fase de floración y fructificación la formulación química Besacote ${ }^{\circledR}$ usada al 100\% (75 kg ha-1) resultó en el incremento del peso, número y diámetro ecuatorial de frutos. Pillimue et al. (1998), en plántulas de tomate de árbol, encontraron que el déficit de $\mathrm{N}$ reduce el área foliar, el peso de la hoja, el peso seco de raíz y la parte aérea. De mismo modo, los autores señalaron que las deficiencias de N, P, K fomentan una sintomatología particular a los 30 ddt (días después del trasplante), como: clorosis generalizada en hojas del estrato inferior y reducción de la tasa de crecimiento.

Debido a la importancia económica del tomate de árbol en Colombia y su elevado potencial en el mercado, es ineludible ampliar los estudios de este cultivo en el área de la nutrición. Por lo anterior, el objetivo de este trabajo consistió en evaluar el efecto de dosis altas y bajas de N, P, K sobre el crecimiento del tomate de árbol en la etapa vegetativa bajo condiciones de invernadero.

\section{MATERIALES Y MÉTODOS}

\section{Localización}

La investigación se realizó en la Facultad de Ciencias Agrarias de la Universidad Nacional de Colombia, sede Bogotá, bajo condiciones de cubierta plástica, con valores promedio de: temperatura del aire de $17,4^{\circ} \mathrm{C}$, humedad relativa de $71,5 \%$ y radiación fotosintéticamente activa (PAR) promedio de 693,5 $\mu \mathrm{mol}$ de fotones $\mathrm{m}^{-2} \mathrm{~s}^{-1}$ (empleando un medidor espectrómetro LI-189, Li-Cor, Lincoln, NE, USA).

\section{Material vegetal}

Se utilizaron plántulas de tomate de árbol (Solanum betaceum Cav.), obtenidas a partir de semilla, ecotipo Rojo Común de tres meses de edad, con promedio de tres hojas verdaderas. Estas fueron trasplantadas en bolsas negras plásticas $(0,80 \times 0,80 \mathrm{~m}$, calibre 6$)$ con contenido de $30 \mathrm{~kg}$. Las plantas trasplantadas se dispusieron bajo invernadero a una distancia de $3 \mathrm{~m}$ entre surcos y 3 m entre plantas. En este experimento solo se evaluó la etapa vegetativa de desarrollo durante $150 \mathrm{ddt}$.

\section{Tratamientos de fertilización}

Se implementó un diseño de bloques completamente aleatorio, con ocho tratamientos y tres réplicas por tratamiento. i) Control comercial (CC): dosis comercial reportada para cultivo de tomate de árbol (Fischer, 2012) y ajustada para el presente experimento (Tab. 2) con base en el análisis fisicoquímico del suelo

Tabla 1. Análisis fisicoquímico del suelo utilizado para los diferentes tratamientos de nutrición mineral, proveniente del municipio del Rosal, Cundinamarca.

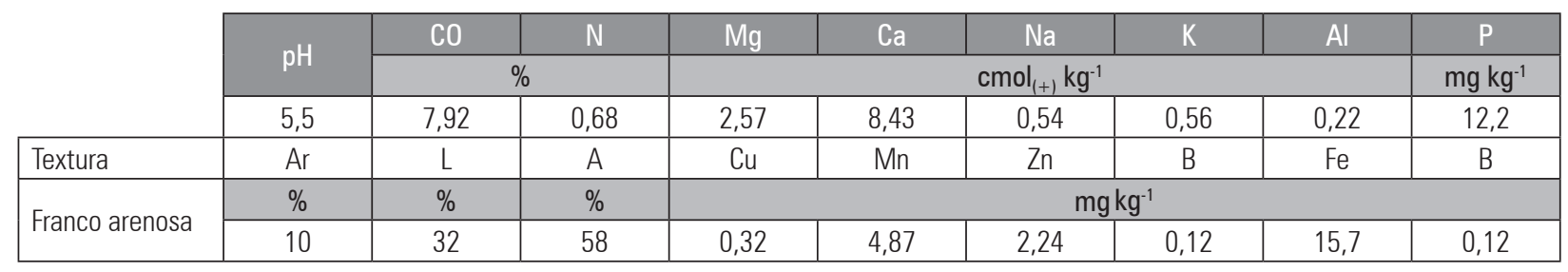

CO: materia orgánica, franco arenoso; Ar: arcilloso; L: limoso; A: arenoso.

El análisis fisicoquímico del suelo fue realizado según la metodología IGAC (2006). 
utilizado (Tab. 1). ii) Control absoluto (CS): plantas sembradas en suelo sin aplicación de ningún nutriente mineral. iii) Bajo N: el cual se aplicó al 10\% (4,12 kg $\left.\mathrm{ha}^{-1}\right)$ por debajo de la dosis comercial $\left(41,2 \mathrm{~kg} \mathrm{ha}^{-1}\right), \mathrm{y}$ el resto de los elementos ( $\mathrm{K}, \mathrm{P}, \mathrm{Mg}, \mathrm{Ca}, \mathrm{S}, \mathrm{B}, \mathrm{Zn}, \mathrm{Mn}$, $\mathrm{Mo}, \mathrm{Fe}, \mathrm{Cl}, \mathrm{Cu})$ se aplicaron con base en la dosis comercial. iv) Bajo P: el cual se aplicó al 10\% (1,18 $\left.\mathrm{kg} \mathrm{ha}^{-1}\right)$ por debajo del control comercial $\left(11,76 \mathrm{~kg} \mathrm{ha}^{-1}\right)$ y el resto de los elementos con base en la dosis comercial. v) Bajo K: el cual se aplicó al 10\% (4,12 $\left.\mathrm{kg} \mathrm{ha}^{-1}\right)$ por debajo del control comercial $\left(41,6 \mathrm{~kg} \mathrm{ha}^{-1}\right)$ y el resto de los elementos con base en la dosis comercial. vi) Alto $\mathrm{N}$ : el cual se aplicó al 200\% (82,3 $\left.\mathrm{kg} \mathrm{ha}^{-1}\right)$ por encima del control comercial, y el resto de los elementos con base en la dosis comercial. vii) Alto P: el cual se aplicó al $200 \%\left(23,52 \mathrm{~kg} \mathrm{ha}^{-1}\right)$ por encima del control y el resto de los elementos con base en la dosis comercial. viii) Alto K: el cual se aplicó al $200 \%\left(82,3 \mathrm{~kg} \mathrm{ha}^{-1}\right)$ por encima del control comercial y el resto de los elementos con base en la dosis comercial (Tab. 2).

El suelo utilizado en este ensayo presentó las siguientes características iniciales: $\mathrm{pH}$ altamente ácido, carbono orgánico (\%) ideal, CIC $\left(\mathrm{cmol}_{(+)} \mathrm{kg}^{-1}\right)$ baja, contenidos bajos de $\mathrm{P}, \mathrm{Mn}, \mathrm{Zn}$, Fe y Cu y altos niveles de Ca, Mg y K (Tab. 1).

Después de los $7 \mathrm{~d}$ de trasplante (etapa para aclimatación) en bolsa, se realizó la aplicación de los tratamientos cada $45 \mathrm{~d}$ (según prácticas en campo) durante la etapa vegetativa, aplicando el fertilizante al suelo, en forma de corona alrededor del tallo de la planta, a $20 \mathrm{~cm}$ de distancia y posteriormente se incorporó en los primeros centímetros del suelo. Las mediciones fisiológicas y muestreos destructivos (crecimiento) se realizaron así: 0 (posterior a la aplicación de tratamientos), 15, 30, 60, 105 y 150 ddt y después de la aplicación de los tratamientos (Tab. 2). Las fuentes de fertilizantes fueron Urea 46-0-0 (Monómeros, Colombo Venezolanos), Nitrato de Calcio 15-0-0-26 (Yara, Colombia), DAP -Fosfato diamónico 18-46-0 (Yara, Noruega), KCl 0-0-60 (Cloruro de Potasio Yara, Noruega), Nutricomplet ${ }^{\circledR} \mathrm{B}, \mathrm{Mg}, \mathrm{Fe}, \mathrm{Cu}, \mathrm{Mo}, \mathrm{S}, \mathrm{Zn}$, $\mathrm{Mn}$ (Ingeplant, Colombia) y Octoborato de Sodio Cosmo-Ion Boro ${ }^{\circledR}$ (Cosmoagro, Colombia). Las plantas se regaron tres veces por semana a capacidad de campo, y se realizó el respectivo manejo agronómico del cultivo, tal como eliminación de arvenses, control de enfermedades y plagas con agroquímicos.

\section{Variables evaluadas}

\section{Parámetros de crecimiento}

Para la medición de los diferentes parámetros, se seleccionaron aleatoriamente tres plantas en cada momento del muestreo.

Número de hojas (NH) y longitud total de la planta (LT): A los 150 ddt se registró con un fluxómetro (Stanley ${ }^{\circledR}$, New Britain, CT) la altura de planta desde el ápice de la raíz principal hasta el ápice del tallo de la planta. Se contabilizó número de hojas verdes completamente expandidas y fotosintéticamente activas.

Área foliar (AF): El área foliar se obtuvo a los 150 ddt, mediante el registro fotográfico de las hojas y con ayuda del programa Image $J^{\oplus}$ v. 1.50i (National Institutes of Health, Bethesada, MD, USA).

Materia seca de órganos (MS): A los $150 \mathrm{ddt}$ se seleccionaron tres (3) plantas por tratamiento, la materia seca (MS) se determinó mediante secado de los órganos (hojas, tallo y raíces) en estufa de secado de

Tabla 2. Tratamientos de fertilización evaluados durante la etapa vegetativa en plantas de tomate de árbol ( $\mathrm{kg} \mathrm{ha}^{-1}$ por ciclo) con una densidad de 880 plantas/ha.

\begin{tabular}{|l|c|c|c|c|c|c|c|c|c|c|c|}
\hline \multicolumn{1}{|c|}{ Tratamientos } & $\mathrm{N}$ & $\mathrm{P}$ & $\mathrm{K}$ & $\mathrm{Ca}$ & $\mathrm{Mg}$ & $\mathbf{B}$ & $\mathrm{Cu}$ & $\mathbf{S}$ & $\mathbf{Z n}$ & $\mathrm{Fe}$ & $\mathrm{Mn}$ \\
\hline CC (control fertilización comercial) & 41,16 & 11,76 & 41,16 & 10,29 & 5,88 & 1,76 & 0,02 & 1,76 & 0,04 & 0,02 & 0,15 \\
\hline CS (control sin fertilizantes) & 0 & 0 & 0 & 0 & 0 & 0 & 0 & 0 & 0 & 0 & 0 \\
\hline Bajo N & 4,12 & 11,76 & 41,16 & 10,29 & 5,88 & 1,76 & 0,02 & 1,76 & 0,04 & 0,02 & 0,15 \\
\hline Bajo P & 41,16 & 1,18 & 41,16 & 10,29 & 5,88 & 1,76 & 0,02 & 1,76 & 0,04 & 0,02 & 0,15 \\
\hline Bajo K & 41,16 & 11,76 & 4,12 & 10,29 & 5,88 & 1,76 & 0,02 & 1,76 & 0,04 & 0,02 & 0,15 \\
\hline Alto N & 82,32 & 11,76 & 41,16 & 10,29 & 5,88 & 1,76 & 0,02 & 1,76 & 0,04 & 0,02 & 0,15 \\
\hline Alto P & 41,16 & 23,52 & 41,16 & 10,29 & 5,88 & 1,76 & 0,02 & 1,76 & 0,04 & 0,02 & 0,15 \\
\hline Alto K & 41,16 & 11,76 & 82,32 & 10,29 & 5,88 & 1,76 & 0,02 & 1,76 & 0,04 & 0,02 & 0,15 \\
\hline
\end{tabular}


aire circulante a $72^{\circ} \mathrm{C}$ durante $120 \mathrm{~h}$ y posteriormente se realizó pesaje en balanza analítica de precisión de cuatro cifras decimales.

\section{Tasas de crecimiento}

Para obtener las tasas de crecimiento se realizaron muestreos destructivos a los 0,15, 30, 60, 105 y 150 ddt. Se emplearon las ecuaciones para estimar las tasas de crecimiento (Gardner et al., 1990) en las plantas de tomate de árbol en etapa vegetativa, como:

Tasa de crecimiento del cultivo (TCC): $\left.1 /(\mathrm{AS})^{*}\left[\left(\mathrm{P}_{2}-\mathrm{P}_{1}\right) / \mathrm{T}_{2}-\mathrm{t}_{1}\right)\right]^{2}$

Tasa de asimilación neta (TAN): $\left.\left[\left(\mathrm{P}_{2}-\mathrm{P}_{1}\right) / \mathrm{T}_{2}-\mathrm{t}_{1}\right)\right]^{*}\left[\left(\mathrm{LnAF}_{2}-\mathrm{LnAF}_{1}\right) /\left(\mathrm{AF}_{2}-\mathrm{AF}_{1}\right)\right]$

Tasa relativa de crecimiento (TRC): $\left(\operatorname{LnP}_{2}-\operatorname{LnP}_{1}\right) /\left(\mathrm{T}_{2}-\mathrm{t}_{1}\right)$

Índice de área foliar (IAF): $\left.\left.\mathrm{AF}_{2}+\mathrm{AF}_{1}\right) / 2\right]^{*}(1 / \mathrm{AS})$

Tasa absoluta de crecimiento (TAC): $\left(\mathrm{P}_{2}-\mathrm{P}_{1}\right) /\left(\mathrm{T}_{2}-\mathrm{t}_{1}\right)$

donde AS: área de suelo $\left(\mathrm{m}^{-2}\right) ; \mathrm{P}_{2}$ : peso seco final de la planta $(\mathrm{g}) ; \mathrm{P}_{1}$ : peso seco inicial de la planta $(\mathrm{g}) ; \mathrm{T}_{2}$ : tiempo final (días); $\mathrm{t}_{1}$ : tiempo inicial (días); $\mathrm{AF}_{2}$ : área foliar final $\left(\mathrm{cm}^{-2}\right) ; \mathrm{AF}_{1}$ : área foliar inicial $\left(\mathrm{cm}^{-2}\right)$.

\section{Análisis estadístico}

Se evaluaron los supuestos de normalidad y homogeneidad de varianzas por medio del paquete estadístico SAS v.8 (SAS Institute, Cary, NC, USA). Posteriormente se realizó un ANAVA y las diferencias obtenidas entre los tratamientos se establecieron a través de la prueba de Tukey $(P \leq 0,05)$ Se usó el paquete estadístico SAS (Statistical Analysis System), v. 8 (Hill y Lewicki, 2007).

\section{RESULTADOS Y DISCUSIÓN}

\section{Área foliar}

Se observó una disminución significativa del área foliar (AF) en los tratamientos de las plantas sin fertilización (CS) $\left(2.668,6 \mathrm{~cm}^{2}\right)$ y Alto P $\left(3.083 \mathrm{~cm}^{2}\right)$, a diferencia de las plantas con el tratamiento que contenía Bajo K $\left(9.488,2 \mathrm{~cm}^{2}\right)$, el cual exhibió el valor más alto de AF a los 150 ddt (Tab. 3). El tratamiento Bajo K incrementó las variables de crecimiento en las plantas de tomate de árbol en comparación de la dosis comercial (CC) y de los otros tratamientos evaluados (Tab. 3). Clavijo-Sánchez et al. (2015), concluyeron que las aplicaciones de K en el cultivo de tomate de árbol mejoran la respuesta de las plantas frente a condiciones de estrés, aumentando los parámetros de crecimiento y la acumulación de biomasa.

\section{Número de hojas y longitud total}

El número de hojas $(\mathrm{NH})$ se presentó en el tratamiento sin fertilización (CS) $(13,3)$ en comparación al valor más alto obtenido con el tratamiento Bajo en K (27) (Tab. 3). Pese a que para la variable longitud total de la planta (LT) no se evidenciaron diferencias estadísticamente significativas, las plantas bajo el tratamiento Bajo en K tienden a presentar la mayor LT a los $150 \mathrm{ddt}$, a diferencia de los demás tratamientos evaluados.

Probablemente, bajo las condiciones del presente estudio las plantas de tomate de árbol en fase vegetativa incrementan el crecimiento con bajo suministro de K (10\%). Malvi (2011) concluyó que al adicionar menores dosis de potasio al sustrato se disminuye la cantidad de $\mathrm{Ca}$ (cuando el análisis de suelo lo reporta alto, como sucede en el presente estudio) e incrementa en menor grado el magnesio (Malvi, 2011), lo que se sugiere que se estimula el incremento de la actividad del K. Por su parte, al estar disponible el K en la planta, eleva la tasa de división y expansión de células meristemáticas (Marschner, 2012).

\section{Materia seca (MS)}

A los 150 ddt la materia seca en hojas (MSH) decreció significativamente en las plantas con Alto P (18,7 g) y CS (16,1 g), sin embargo, el tratamiento Bajo K registró un aumento significativo (61,9 g) (Tab. 3). La materia seca de tallos (MST) se redujo significativamente para las plantas sometidas a Alto P, CS y Alto $\mathrm{K}(28,8,34,1$ y $70,3 \mathrm{~g}$, respectivamente), a diferencia de las plantas con Bajo K las cuales registraron el mayor valor (109,8 g). De mismo modo, se observó una disminución significativa de la materia seca de raíces (MSR) en plantas bajo el control suelo (CS) del 76\% a diferencia de las plantas con Alto K. En general, la materia seca total (MS total) registró una disminución 
Tabla 3. Efecto de diferentes dosis de $\mathrm{N}, \mathrm{P}$ y $\mathrm{K}$ en los parámetros de crecimiento de tomate de árbol a los $150 \mathrm{ddt}$.

\begin{tabular}{|c|c|c|c|c|c|c|c|}
\hline & $\mathrm{AF}\left(\mathrm{cm}^{2}\right)$ & NH & LT $(\mathrm{cm})$ & MSH (g) & MST (g) & MSR (g) & MS total $(\mathrm{g})$ \\
\hline $\operatorname{Pr}>\mathrm{F}$ & 0,0046 & 0,0489 & 0,0790 & 0,0073 & 0,0153 & 0,0276 & 0,0024 \\
\hline CC & $\begin{array}{c}4.859,7 \pm 680 \\
a b\end{array}$ & $\begin{array}{c}21,7 \pm 2,8 \\
a b\end{array}$ & $\begin{array}{c}190 \pm 4,6 \\
a\end{array}$ & $\begin{array}{c}40,6 \pm 0,3 \\
a b\end{array}$ & $\begin{array}{c}62,6 \pm 5,0 \\
a b\end{array}$ & $\begin{array}{c}141,9 \pm 17,3 \\
a b\end{array}$ & $\begin{array}{c}245,1 \pm 22,3 \\
a b c\end{array}$ \\
\hline CS & $\begin{array}{c}2.668,6 \pm 276,9 \\
b\end{array}$ & $\begin{array}{c}13,3 \pm 0,7 \\
b\end{array}$ & $\begin{array}{c}154,7 \pm 5,5 \\
a\end{array}$ & $\begin{array}{c}16,1 \pm 0,8 \\
b\end{array}$ & $\begin{array}{c}34,1 \pm 5,0 \\
b\end{array}$ & $\begin{array}{c}63,9 \pm 3,7 \\
b\end{array}$ & $\begin{array}{c}114,1 \pm 1,9 \\
c\end{array}$ \\
\hline Bajo N & $\begin{array}{c}6.748,7 \pm 31,6 \\
a b\end{array}$ & $\begin{array}{c}22,3 \pm 0,7 \\
a b\end{array}$ & $\begin{array}{c}201,7 \pm 8,3 \\
a\end{array}$ & $\begin{array}{c}44,1 \pm 4,5 \\
a b\end{array}$ & $\begin{array}{c}73,2 \pm 6,1 \\
a b\end{array}$ & $\begin{array}{c}106,3 \pm 13,4 \\
a b\end{array}$ & $\begin{array}{c}223,6 \pm 5,5 \\
\text { abc }\end{array}$ \\
\hline Alto $\mathrm{N}$ & $\begin{array}{c}8.135,0 \pm 1736,1 \\
a b\end{array}$ & $\begin{array}{c}27,3 \pm 3,9 \\
a b\end{array}$ & $\begin{array}{c}159,7 \pm 12,6 \\
a\end{array}$ & $\begin{array}{c}49,9 \pm 10,6 \\
a b\end{array}$ & $\begin{array}{c}167 \pm 153 \\
a b\end{array}$ & $\begin{array}{c}119 \pm 35,8 \\
a b\end{array}$ & $\begin{array}{c}336,1 \pm 144,5 \\
a b\end{array}$ \\
\hline Bajo P & $\begin{array}{c}6.162,0 \pm 176,3 \\
a b\end{array}$ & $\begin{array}{c}23,0 \pm 3,3 \\
a b\end{array}$ & $\begin{array}{c}196,3 \pm 11,2 \\
a \\
\end{array}$ & $\begin{array}{c}46,4 \pm 6,7 \\
a b\end{array}$ & $\begin{array}{c}70,9 \pm 5,2 \\
a b\end{array}$ & $\begin{array}{c}206,5 \pm 42 \\
a b\end{array}$ & $\begin{array}{c}323,8 \pm 40,4 \\
a b\end{array}$ \\
\hline Alto $P$ & $\begin{array}{c}3.083 \pm 212,6 \\
b\end{array}$ & $\begin{array}{c}15,3 \pm 0,7 \\
a b\end{array}$ & $\begin{array}{c}154,7 \pm 10,8 \\
a\end{array}$ & $\begin{array}{c}18,7 \pm 0,8 \\
b\end{array}$ & $\begin{array}{c}28,8 \pm 4,4 \\
b\end{array}$ & $\begin{array}{c}99,7 \pm 13,6 \\
a b\end{array}$ & $\begin{array}{c}147,2 \pm 10,4 \\
\text { bc }\end{array}$ \\
\hline Bajo K & $\begin{array}{c}9.488,2 \pm 413,2 \\
a\end{array}$ & $\begin{array}{c}27,0 \pm 0,5 \\
a\end{array}$ & $\begin{array}{c}256 \pm 4,9 \\
a\end{array}$ & $\begin{array}{c}61,9 \pm 0,1 \\
a\end{array}$ & $\begin{array}{c}109,8 \pm 15,3 \\
a\end{array}$ & $\begin{array}{c}144,9 \pm 24,9 \\
a b\end{array}$ & $\begin{array}{c}316,6 \pm 34,8 \\
a b\end{array}$ \\
\hline Alto $\mathrm{K}$ & $\begin{array}{c}6.205,2 \pm 1375,4 \\
a b\end{array}$ & $\begin{array}{c}19,3 \pm 2,0 \\
a b\end{array}$ & $194,3 \pm 10,9 a$ & $\begin{array}{c}39,4 \pm 9,3 \\
a b\end{array}$ & $\begin{array}{c}70,3 \pm 17,5 \\
a b\end{array}$ & $271,3 \pm 55,6 a$ & $\begin{array}{c}381,0 \pm 64 \\
a\end{array}$ \\
\hline
\end{tabular}

CC: control comercial; CS: control sin aplicación de fertilizantes; Bajo N: nitrógeno al 10\% de la dosis recomendada (CC); Bajo P: fósforo al 10\% de CC; Bajo K: potasio al 10\% de CC; Alto N: nitrógeno al 200\% de CC; Alto P: fósforo al 200\% de CC; Alto K: potasio al 200\% de CC.

AF: área foliar; NH: número de hojas; LT: longitud total; MSH: masa seca de hojas; MST: masa seca de tallo; MSR: masa seca de raíces; MS total: masa seca total. Promedios \pm error estándar. $n=3$. Promedios con letras distintas indican diferencia significativa según la prueba de Tukey $(P \leq 0,05)$.

en plantas sin suministro de fertilizantes (CS) $(114,1$ g) y el mayor valor lo presentaron las plantas con Alto K (381 g) (Tab. 3).

El tratamiento CS presentó una disminución en la materia seca (hojas, tallo, raíces y total), número de hojas, área foliar y longitud total, además de sintomatología asociada a deficiencia de $\mathrm{N}$ (clorosis total de los foliolos del tercio bajo), $\mathrm{K}$ (clorosis en el margen de las hojas y en algunos casos necrosis en el borde de los foliolos del tercio bajo) y $\mathrm{Mg}$ (clorosis intervenal en el tercio inferior de la planta, quedando un tono de verde más fuerte en las venas de la hoja). Lo anterior, coincide con las bajas cantidades encontradas en el análisis de suelo del presente estudio. Elementos como N, K, P y Mg presentan alta demanda en etapa vegetativa para promover el desarrollo y crecimiento de las plantas (Giorgi et al., 2009). En el presente ensayo se evidenció que las plantas del control suelo sin fertilizantes (CS) exhibieron sintomatología asociada a deficiencia de nitrógeno. La carencia o limitación de $\mathrm{N}$ en la planta ocasionado por la no adición de fertilizantes, altera el balance de hormonas a causa de la interrupción de la exportación de citoquininas desde la raíz hasta los brotes, disminución en la síntesis de proteínas, déficit en la actividad enzimática y disminución del crecimiento (Rubio et al., 2009; Baloch et al., 2014).

Por otro lado, al adicionar bajas cantidades de $\mathrm{K}$ no se afectó el crecimiento ni las variables fisiológicas evaluadas en plantas de tomate de árbol. Lo anterior, es probablemente debido a que se presenta la condición de una parcial sustitución del $\mathrm{K}^{+}$con el sodio $\left(\mathrm{Na}^{+}\right)$, a razón de la escasa presencia del macroelemento en el suelo utilizado; donde las funciones tales como elongación celular y transporte de cationes a larga distancia son sustituidas o potencializadas por el sodio (Subbarao et al., 2003).

\section{Índices de crecimiento}

\section{Índice de área foliar (IAF)}

Las plantas del tratamiento Bajo $\mathrm{K}$ aumentaron el IAF a través del tiempo en comparación con los otros tratamientos evaluados, alcanzando el mayor valor de entre los tratamientos $(15,039)$ a los $150 \mathrm{ddt}$ (Fig. 1A). Asimismo, las plantas bajo los tratamientos Alto $\mathrm{N}$ y Bajo $\mathrm{N}$ presentaron un valor máximo de 12,9 y 10,6 , respectivamente a los $150 \mathrm{ddt}$, seguido por Alto 
K presentando un valor de 9,87 (Figs. 1A, B). Laisk et al. (2005) indicaron que al momento de producir más hojas, las hojas de estratos bajos quedan sombreadas y la fotosíntesis se reduce en relación con la radiación. Del mismo modo, se evidenció que las plantas con Bajo K alcanzaron los parámetros de crecimiento más elevados como el AF y NH (Tab. 3), lo que probablemente es debido a la menor necesidad o demanda de este elemento en la fase vegetativa. En los parámetros de crecimiento, en todos los tratamientos evaluados entre los 50 y $150 \mathrm{ddt}$ posiblemente se presentó una reducción en la velocidad de asimilación neta de fotoasimilados (dato no medido en el presente experimento).

\section{Tasa absoluta de crecimiento (TAC)}

A partir de los 100 ddt se registró un incremento en la ganancia de biomasa por día (TAC) de plantas con Alto K (Fig. 1D), seguido por Bajo K, Bajo P y CC (Fig. 1C). A los $150 \mathrm{ddt}$ las plantas sometidas a los tratamientos Alto K, Bajo K, Bajo P y CC presentaron el mayor valor $\left(10,4,7,7,5,1,3,7 \mathrm{~g} \mathrm{~g}^{-1} \mathrm{~d}^{-1}\right.$, respectivamente), a diferencia de las plantas con Alto $\mathrm{N}$ y Alto
$\mathrm{P}\left(0,16\right.$ y $0,19 \mathrm{~g} \mathrm{~g}^{-1} \mathrm{~d}^{-1}$ a los $147 \mathrm{ddt}$, respectivamente) (Fig. 1D). Se evidenció un decrecimiento de la TAC para las plantas con los tratamientos Alto N, Alto P y CS $\left(1,77,2,21\right.$ y $\left.0,97 \mathrm{~g} \mathrm{~g}^{-1} \mathrm{~d}^{-1}\right)$ (Fig. 1D).

\section{Tasa relativa de crecimiento (TRC)}

En general, los tratamientos CC y Bajo K (0,39 y $0,388 \mathrm{~g} \mathrm{~g}^{-1} \mathrm{~d}^{-1}$, respectivamente) alcanzaron la mayor TRC en los primeros días, luego decreció a partir de los $15 \mathrm{ddt}$, finalmente los valores se reducen hasta los $150 \mathrm{ddt}\left(0,015\right.$ y $0,014 \mathrm{~g} \mathrm{~g}^{-1} \mathrm{~d}^{-1}$, respectivamente) (Fig. $1 \mathrm{E})$. Los anteriores resultados coinciden con lo reportado por Almanza et al. (2008) donde sugiere que la TRC presenta un incremento de la curva al inicio del ciclo de las plantas de lulo que se disminuyen a través del ciclo de vida, similar a lo reportado por Barraza (2000).

\section{Tasa de crecimiento del cultivo (TCC)}

La TCC registró un incremento en varios de los tratamientos, acorde avanzó el crecimiento de las plantas. Las plantas con Alto K y Bajo K alcanzaron los valores

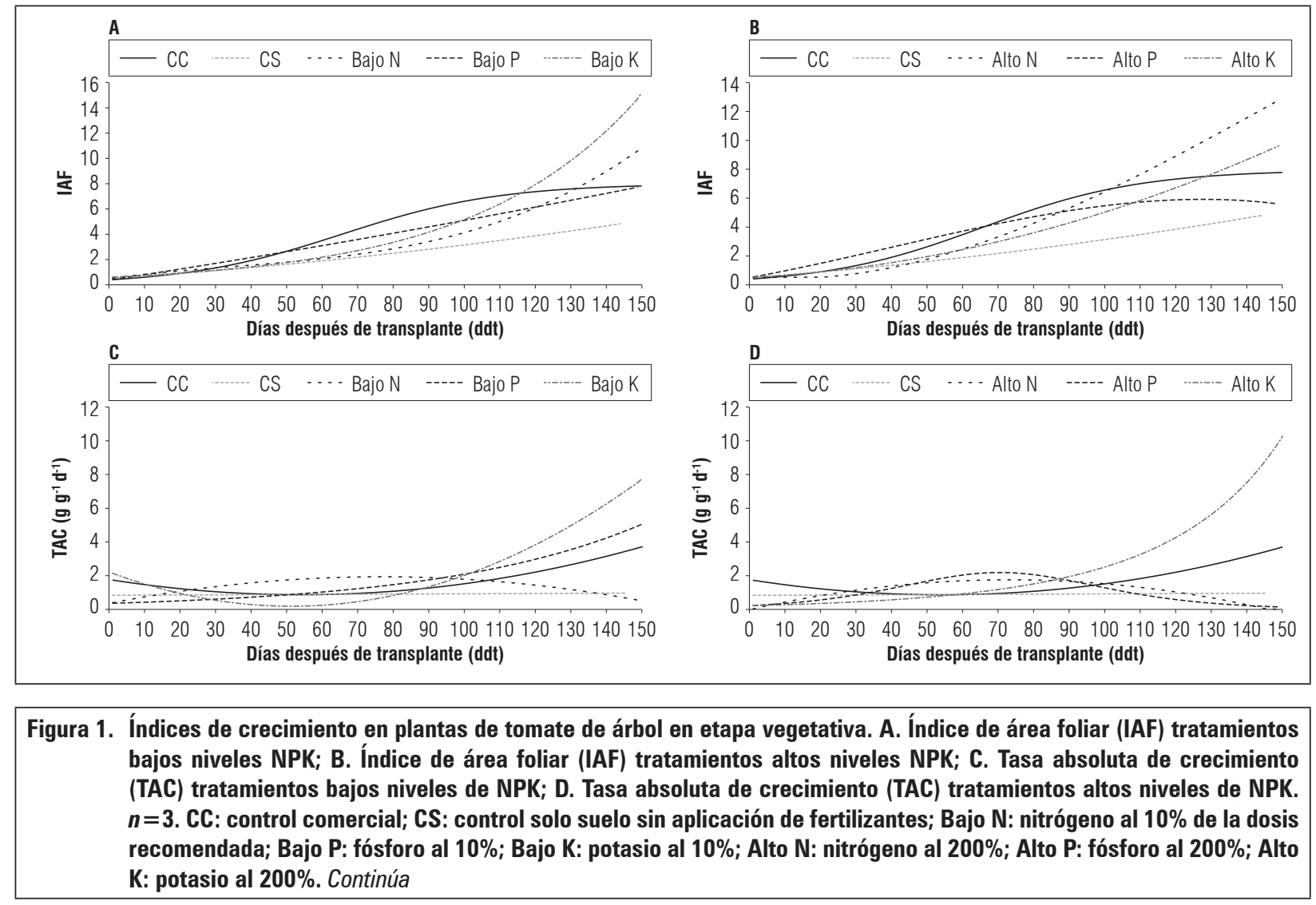




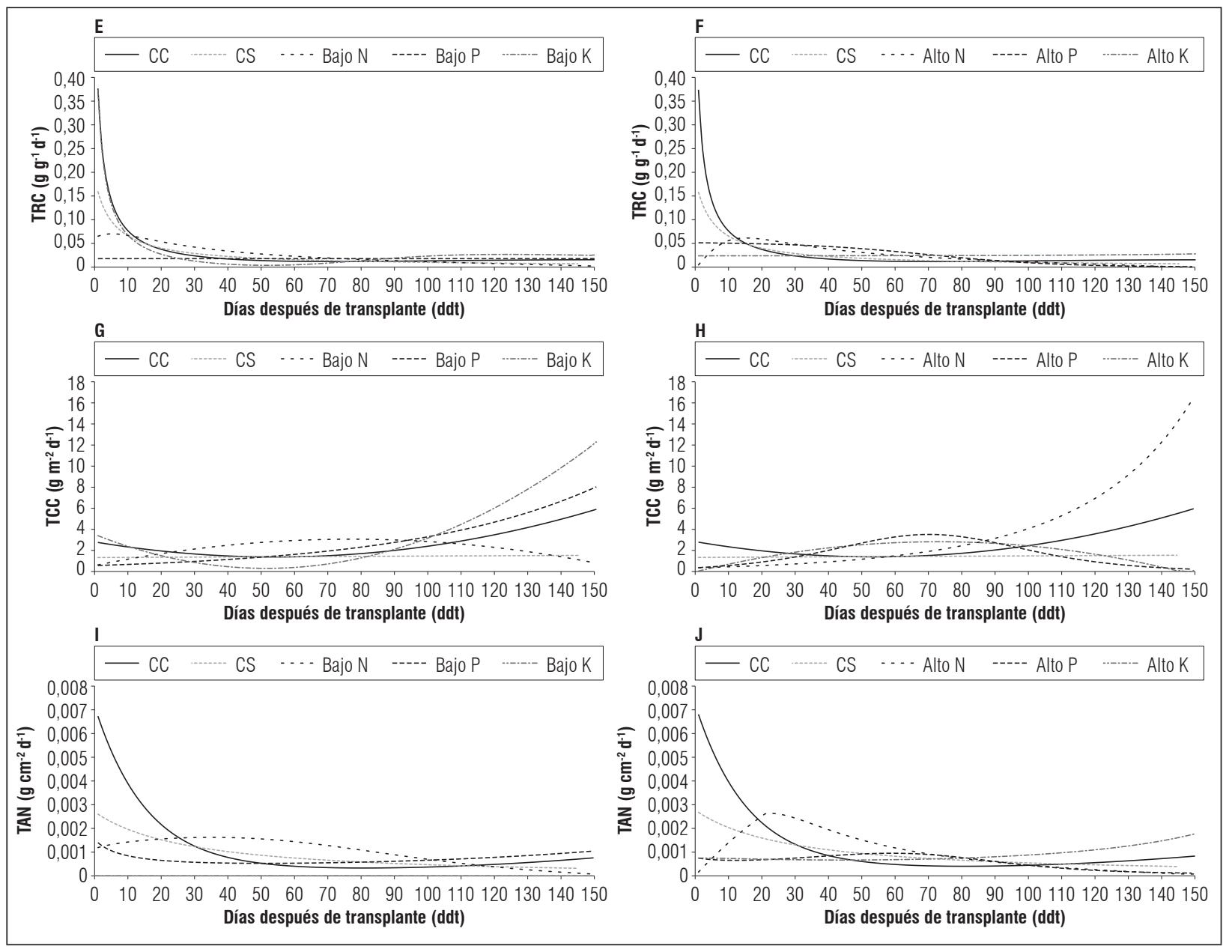

Figura 1. Índices de crecimiento en plantas de tomate de árbol en etapa vegetativa. continuación: $\mathrm{E}$. Tasa relativa de crecimiento (TRC) tratamientos bajos niveles de NPK; F. Tasa relativa de crecimiento (TRC) tratamientos altos niveles de NPK; G. Tasa de crecimiento del cultivo (TCC) tratamientos bajos niveles de NPK; H. Tasa de crecimiento del cultivo (TCC) tratamientos altos niveles de NPK; I. Tasa de asimilación neta (TAN) tratamientos bajos niveles NPK; J. Tasa de asimilación neta (TAN) tratamientos altos niveles NPK. $n=3$. CC: control comercial; CS: control solo suelo sin aplicación de fertilizantes; Bajo N: nitrógeno al 10\% de la dosis recomendada; Bajo P: fósforo al 10\%; Bajo K: potasio al 10\%; Alto N: nitrógeno al $200 \%$; Alto P: fósforo al $200 \%$; Alto K: potasio al $\mathbf{2 0 0} \%$.

más altos (16,61 y 12,42 $\mathrm{g} \mathrm{m}^{-2} \mathrm{~d}^{-1}$, respectivamente) a los 150 ddt (Figs. 1G, H). La TCC más baja se registró a los $150 \mathrm{ddt}$ en plantas con los tratamientos Bajo $\mathrm{N}$ $\left(0,97 \mathrm{~g} \mathrm{~m}^{-2}\right.$ día $\left.{ }^{-1}\right)$, Alto $\mathrm{N}$, Alto P y CS $(0,2,0,5$ y $1,5 \mathrm{~g}$ $\mathrm{m}^{-2}$ día $^{-1}$, respectivamente) (Figs. $1 \mathrm{G}, \mathrm{H}$ ). Posiblemente, Bajo K resulte en una mayor eficiencia fotosintética (variable no medida en esta fase del crecimiento); no obstante, se evidenció que este tratamiento registró el mayor AF (Tab. 3). Barraza (2000) señaló que la TCC y el área de las hojas determinan la efectividad de absorción de la radiación para la generación de fotoasimilados.

\section{Tasa de asimilación neta (TAN)}

Las plantas con el tratamiento Alto K presentaron la mayor ganancia de materia seca por $\mathrm{cm}^{2}$ a los 150 ddt a diferencia de los demás tratamientos (Fig. 1J), lo cual posiblemente se asocia con la ganancia final de biomasa total de la planta (Tab. 3). La disminución en la acumulación de materia seca a través del tiempo, probablemente se relaciona con la menor área foliar expuesta a la radiación debido al mayor sombrío de las hojas (Barraza, 2000; Laisk et al., 2005). 


\section{CONCLUSIONES}

Se encontró bajo las condiciones evaluadas en el ensayo y suelo utilizado, disminución del crecimiento de las plántulas de tomate de árbol frente a la no aplicación de fertilizantes (CS). El tratamiento Bajo K aumentó el AF, NH, LT, MSH, MST, IAF y TRC, y el tratamiento Alto K exhibió la mayor TCC, no obstante, pese a lo encontrado en el presente estudio, no fue concluyente el resultado de Alto y Bajo K, en el aumento de las anteriores variables. De acuerdo a los resultados encontrados en el presente estudio, para suelos y condiciones similares, se sugiere reevaluar las dosis que actualmente se aplican en campo en cultivos comerciales de tomate de árbol, con el fin de mejorar parámetros relacionados con crecimiento.

\section{AGRADECIMIENTOS}

Los autores agradecen a Colciencias por la financiación de esta investigación perteneciente al proyecto "Ecofisiología, nutrición mineral y manejo integrado de plagas y enfermedades en aguacate, curuba, gulupa y tomate de árbol orientados hacia su manejo agronómico, como materia prima para el desarrollo de productos de interés comercial" coordinado por Dra. L.M. Melgarejo, de la Red Nacional para la Bioprospección RIFRUTBIO contrato 459/2013. Al Departamento de Biología y la Facultad de Ciencias Agrarias de la Universidad Nacional de Colombia, sede Bogotá. A la financiación dada dentro del proyecto a Claudia Ramírez para la realización del presente trabajo en su tesis de maestría. Del mismo modo los autores agradecen al Dr. Manuel Iván Gómez de INGEPLANT por facilitar los fertilizantes con microelementos y al Dr. Jaime Torres de la Facultad de Ciencias Agrarias por su valiosa colaboración para definir las dosis de fertilizantes.

Conflicto de intereses: el manuscrito fue preparado y revisado con la participación de los autores, quienes declaran no tener algún conflicto de interés que coloquen en riesgo la validez de los resultados aquí presentados.

\section{REFERENCIAS BIBLIOGRÁFICAS}

Acosta-Quezada, P., M. Raigónb, A. Riofrío-Cuenca, M. García-Martínez, M. Plazas, J. Burneoa, J. Figueroa, S. Vilanovac y J. Prohen. 2015. Diversity for chemical composition in a collection of different varietal types of tree tomato (Solanum betaceum Cav.), an Andean exotic fruit. Food Chem. 169(15), 327-335. Doi: 10.1016/j.foodchem.2014.07.152

Almanza, P., P. Serrano y O. Castro. 2008. Respuesta fisiológica del lulo (Solanun quitoense Lam.) a la fertilización orgánica en Tinjacá, Boyacá. Cultura Científica 6, 83-86.

Armengaud, P., R. Sulpice, A. Miller, M. Stitt, A. Amtmann e Y. Gibon. 2009. Multilevel analysis of primary metabolism provides new insights into the role of potassium nutrition for glycolysis and nitrogen assimilation in Arabidopsis roots. Plant Physiol. 150(2), 772-785. Doi: 10.1104/pp.108.133629

Baloch, P., R. Uddin, F. Nizamani, A. Solangi y A. Siddiqui. 2014. Effect of nitrogen, phosphorus and potassium fertilizers on growth and yield characteristics of radish (Raphanus sativus L.). Am. Eurasian. J. Agric. Environ. Sci. 14(6), 565-569. Doi: 10.5829/idosi. aejaes.2014.14.06.12350

Barraza, F. 2000. Crecimiento del chile manzano (Capsicum pubescens R. y P.) en cuatro soluciones nutritivas bajo invernadero. Tesis de maestría. Instituto de Horticultura, Universidad Autónoma de Chapingo, México DF, México.

Boussadia, O., K. Steppe, H. Zgallai, D. Ben El Hadj, M. Braham, R. Lemeur y M. Van Labeke. 2010. Effects of nitrogen deficiency on leaf photosynthesis, carbohydrate status and biomass production in two olive cultivars 'Meski' and 'Koroneiki'. Sci. Hortic. 123, 336342. Doi: 10.1016/j.scienta.2009.09.023

Clark, C. y A. Richardson. 2002. Biomass and mineral nutrient partitioning in a developing tamarillo (Solanum betaceum) crop. Sci. Hort. 94, 41-51. Doi: 10.1016/ S0304-4238(01)00355-7

Clavijo-Sánchez, N., N. Flórez-Velasco y H. Restrepo-Díaz. 2015. Potassium nutritional status affects physiological response of tamarillo plants (Cyphomandra betacea Cav.) to drought stress. J. Agr. Sci. Tech. 17, 1839-1849.

Divito, G. y V. Sadras. 2014. How do phosphorus, potassium and sulphur affect plant growth and biological nitrogen fixation in crop and pasture legumes? A meta-analysis. Field Crops Res. 156, 161-171. Doi: 10.1016/j.fcr.2013.11.004

Fischer, G. (ed.). 2012. Manual para el cultivo de frutales en el trópico. Produmedios, Bogotá, Colombia.

Gardner, F., R. Pearce y R. Mitchell. 1990. Physiology of crop plants. 2 a ed. Iowa State University, Ames, IA, USA.

Giorgi, A., M. Mingozzi, M. Madeo, G. Speranza y M. Cocucci. 2009. Effect of nitrogen starvation on the phenolic metabolism and antioxidant properties of yarrow (Achillea collina Becker ex Rchb.). Food Chem. 114(1), 204-211. Doi: 10.1016/j.foodchem.2008.09.039

Hill, T. y P. Lewicki. 2007. Statistics: methods and applications. StatSoft, Tulsa, OK, USA. 
Hu, W., X. Lv, J. Yang, B. Chen, W. Zhao, Y. Meng, Y. Wang, Z. Zhou y D. Oosterhuis. 2016. Effects of potassium deficiency on antioxidant metabolism related to leaf senescence in cotton (Gossypium hirsutum L.). Field Crops Res. 191, 139-149. Doi: 10.1016/j. fcr.2016.02.025

IGAC (Instituto Geográfico Agustín Codazzi). 2006. Métodos analíticos de laboratorio de suelos. 6ª ed. Bogotá, Colombia.

Kanai, S., R. Moghaieb, R. El-Shemy, R. Panigrahi, R. Mohapatra, J. Ito, N. Nguyen, H. Saneoka y K. Fujita. 2011. Potassium deficiency affects water status and photosynthetic rate of the vegetative sink in greenhouse tomato prior to its effects on source activity. Plant Sci. 180, 368-374. Doi: 10.1016/j.plantsci.2010.10.011

Kalaji, H., A. Oukarroum, V. Alexandrov, V. Kouzmanova, M. Brestic, M. Zivcak, I. Samborska, M. Cetner, S. Allakhverdiev y V. Goltsev. 2014. Identification of nutrient deficiency in maize and tomato plants by in vivo chlorophyll a fluorescence measurements. Plant Physiol. Biochem. 81, 16-25. Doi: 10.1016/j. plaphy.2014.03.029

Laisk, A., H. Eichelmann, V. Oja, B. Rasulov, E. Padu, I. Bichele, H. Pettai y O. Kull. 2005. Adjustment of leaf photosynthesis to shade in a natural canopy: rate parameters. Plant Cell Environ. 28, 375-388. Doi: 10.1111/j.1365-3040.2004.01274.x

Malvi, R. 2011. Interaction of micronutrients with major nutrients with special reference to potassium. Karnataka J. Agric. Sci. 24(1), 106-109.

Marschner, H. 2012. Mineral nutrition of higher plants. 3rd ed. Academia Press, Londres, UK.

Martínez, F., J. Sarmiento, G. Fischer y F. Jiménez. 2008. Efecto de la deficiencia de N, P, K, Ca, Mg y B en componentes de producción y calidad de la uchuva (Physalis peruviana L.). Agron. Colomb. 26(3), 389-398.

Montalvo, G. 2010. Evaluación de dos formulaciones químicas a base de N, P, K para el desarrollo y crecimiento de plantas de tomate de árbol (Solanum betaceum). Tesis de pregrado. Escuela Superior Politecnica de Chimborazo, Riobamba, Ecuador.

Oosterhuis, D., D. Loka, E. Kawakami y W. Pettigrew. 2014. The physiology of potassium in crop production. Adv. Agron. 126, 203-233. Doi: 10.1016/ B978-0-12-800132-5.00003-1

Osorio, C., N. Hurtado, C. Dawid, T. Hofmann, F. Heredía y A. Morales. 2012. Chemical characterisation of anthocyanins in tamarillo (Solanum betaceum Cav.) and Andes berry (Rubus glaucus Benth.) fruits. Food Chem. 132, 1915-1921. Doi: 10.1016/j.foodchem.2011.12.026

Pilco, J. 2009. Evaluación de dos formulaciones químicas a base de N, P, K para floración y reproducción de plantas de tomate de árbol (Solanum betaceum). Trabajo de pregrado. Escuela Superior Politécnica de Chimborazo. Riobamba, Ecuador.

Pillimue, G., N. Barrera y E. Cantillo. 1998. Determinación de deficiencias de elementos mayores en plántulas de tomate de árbol Solanum betaceum sinónimo Solanum betaceum (Cav.) Sendt. Acta Agron. 48(3), 62-67.

Rios, J., S. Jaramillo, L. Gonzalez y J. Cortes. 2010. Determinación del efecto de diferentes niveles de fertilización en papa (Solanum tuberosum ssp. Andigena) Diacol Capiro en un suelo con propiedades ándicas de Santa Rosa de Osos, Colombia. Rev. Fac. Nal. Agr. Medellín 63(1), 5225-5237 Doi: 10.15446/rfnam

Rubio, V., R. Bustos, M. Irigoyen, X. Cardona-López, R. Rojas-Triana y J. Paz-Ares. 2009. Plant hormones and nutrient signaling. Plant Mol. Biol. 69(4), 361-373. Doi: 10.1007/s11103-008-9380-y

Subbarao, G., L. Berry y R. Wheeler. 2003. Sodium - A functional plant nutrient. Crit. Rev. Plant Sci. 22(5), 391416. Doi: 10.1080/07352680390243495

Vitousek, P., S. Porder, B. Houlton y O. Chadwick. 2010. Terrestrial phosphorus limitation: mechanisms, implications, and nitrogen-phosphorus interactions. Ecol. Appl. 20, 5-15. Doi: 10.1890/08-0127.1 